\title{
Sarcoidosis: Association with small bowel disease and folate deficiency
}

\author{
S M MacRury, G McQuaker, R Morton, R Hume
}

\begin{abstract}
A 30 year old woman with recurrent anaemia due to folate deficiency had evidence of sarcoid granuloma on small bowel biopsy but was presumed to have Crohn's disease. The diagnosis of small bowel sarcoidosis was not seriously considered until she developed systemic manifestations of sarcoidosis (cutaneous and pulmonary lesions) over the following 20 years. Sarcoidosis of the gastrointestinal tract, particularly the small bowel, is rare and this case is unusual because bowel pathology preceded more generalised lesions. As far as is known it is also the first case to be described presenting with malabsorption of folic acid.
\end{abstract}

Disease of the small bowel is the least common form of gastrointestinal pathology in sarcoidosis, and to date there have only been six well documented and histologically confirmed cases. $^{1-6}$ In most cases symptomatic small bowel sarcoidosis developed on a background of established disease, usually affecting the lungs, skin, or eyes, ${ }^{2-5}$ although these other manifestations may have occurred several years previously. Disseminated gastrointestinal disease has also been reported in several of these cases $^{13-5}$ often with asymptomatic granulomas in the liver and spleen. ${ }^{1356}$ Symptoms of small bowel disease in these patients have included abdominal pain, diarrhoea, weight loss, and in one patient acute appendicitis. ${ }^{4}$

In some patients, malabsorption has resulted, and a megaloblastic anaemia due to malabsorption of vitamin $B_{12}$ has been documented. This was explained by terminal ileal disease in one patient ${ }^{3}$ and achlorhydria in another, ${ }^{2}$ and in a third patient was attributed to achlorhydria secondary to gastric sarcoidosis. ${ }^{4}$ These symptoms and signs may resemble Crohn's disease of the small bowel, but sarcoidosis of the small bowel generally tends to follow a more benign course and responds to treatment with corticosteroids. We present a case of sarcoidosis affecting the small bowel which presented with recurrent anaemia due to folate deficiency.

\section{Case report}

A 30 year old woman who presented to the outpatient department with a history of fatigue was found to have a severe megaloblastic anaemia (haemoglobin $4.6 \mathrm{~g} / \mathrm{dl}$ ), with features of folate deficiency. The anaemia was thought to be dietary in origin and she responded well to oral supplements of folic acid, $5 \mathrm{mg}$ three times a day, which were continued for one year. Five years later she presented again with anaemia (haemoglobin $6.3 \mathrm{~g} / \mathrm{dl}$ ) due to folate deficiency. There was no history of alcohol abuse and dietary history confirmed adequate intake of all haematinics. Investigation for malabsorption, including faecal fat collection, xylose absorption test, and glucose tolerance tests yielded normal results, as did a barium meal and small bowel follow-through $x$-ray picture. A small bowel biopsy specimen revealed "sarcoid-like" granulomas in the mucosa with partial villous atrophy (fig 1) and a diagnosis of low grade Crohn's disease was suggested.

The patient remained well on long term folic acid supplementation until 10 years later when she presented to the dermatology clinic with an erythematous infiltrative lesion on her face over the right mandibular area, which, when biopsied showed a non-caseating epithelioid granulomatous reaction, including "naked granulomas", throughout the dermis. Fungi and foreign bodies were not identified and the appearances were suggestive of sarcoidosis (fig 2).

The Mantoux test was negative and the Kveim test positive. The angiotensin converting enzyme activity was $47 \mathrm{U} / 1$ (normal range $<88 \mathrm{U} / \mathrm{l}$ ). Four years later she presented with a history of upper abdominal pain and dyspnoea. Barium meal and small bowel followthrough $x$-ray picture showed thickened and irregular mucosal folds in the upper jejunum. The chest $x$-ray picture showed bilateral pulmonary infiltrates in both midzones consistent with sarcoidosis. There was no hilar lymphadenopathy. A gallium scan showed active inflammatory disease of the small bowel and lungs. Pulmonary function tests were normal. Both of these recent symptoms settled spontaneously and the patient declined further investigation.

\section{Discussion}

There are many causes of gastrointestinal granulomas, including tuberculosis, carcinoma, Whipple's disease and foreign body reactions. Both the duration of this patient's illness and her general well-being throughout allows most of these conditions to be excluded. The combination of the histological lesions in the small bowel biopsy specimen, together with the cutaneous and pulmonary lesions, suggests a diagnosis of systemic sarcoidosis with small bowel disease in this patient. The most likely 
Figure 1 Small bowel biopsy specimens showing non-caseating granuloma (arrow) and severe partial villous atrophy.
Figure 2 Biopsy specimen of facial lesion showing several non-caseating granulomata within the dermis.
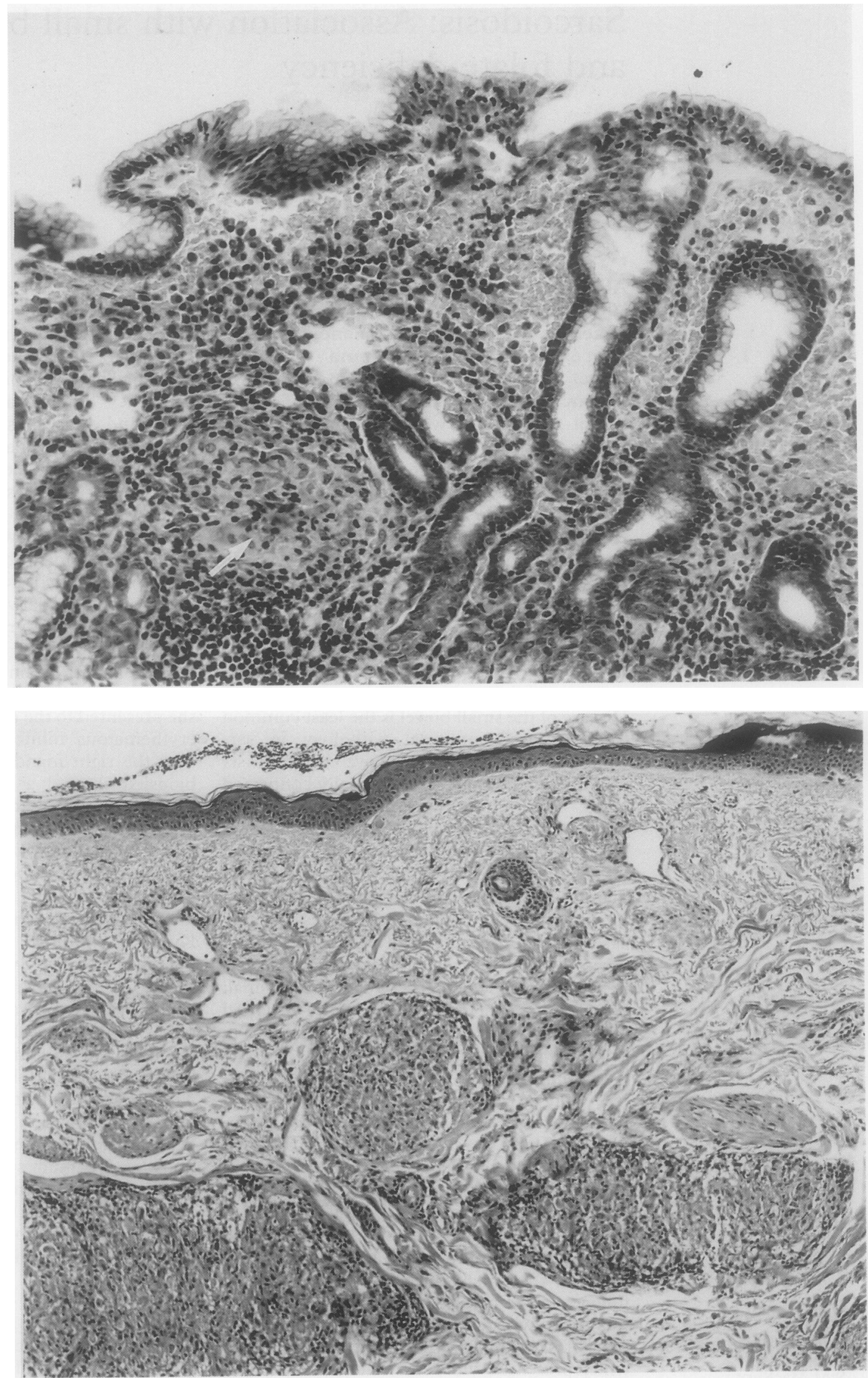

alternative explanation for the intestinal pathology would be Crohn's disease. Crohn's disease, however, affects the entire bowel wall whereas sarcoidosis affects only the mucosa. Thus patients with active Crohn's disease, in addition to presenting with abdominal pain, diarrhoea, and weight loss, are prone to fistulae and stricture formation. The absence of these symptoms and sequelae mitigates against diagnosis of Crohn's disease and suggests a more benign condition like sarcoid. The villous abnormalities described on biopsy explain the patient's folate deficiency, but although similar changes have been described in another patient 
with small bowel sarcoidosis, ${ }^{3}$ the association between intestinal sarcoid and villous atrophy is unknown.

Crohn's disease and sarcoidosis have common systemic manifestations, and the most frequently occurring cutaneous lesion to be associated with both conditions is erythema nodosum. However, cutaneous granulomas do occur in Crohn's disease, though rarely in areas which are not a direct extension from the gastrointestinal tract or of skin apposition. ${ }^{7}$ Respiratory tract disease, in the form of bronchial granulomas, has been reported in Crohn's disease but in the affected patients there was no other evidence of lung disease. ${ }^{8}$

Coexistent Crohn's disease and sarcoidosis has been reported in a few patients, ${ }^{9}$ and because of their histological and immunological similarities it has been postulated that they may represent an overlapping syndrome of granuloma formation due to a generalised host response to various aetiological agents. However, the existence of non-progressive small bowel disease over two decades, together with a positive Kveim test, is much more compatible with sarcoidosis than Crohn's disease. ${ }^{10}$

Given the protean nature of sarcoidosis, the generally benign course, and the occurrence of asymptomatic lesions it could be that intestinal disease is commoner than previously thought. Patients with sarcoidosis who present with persistent diarrhoea, malabsorption, or folate deficiency should be investigated for intestinal sarcoidosis, and the diagnosis should be considered in patients with small bowel pathology who later develop manifestations of systemic sarcoidosis.

1 Miyamoto C, Nomura S, Kudo E, HamamotoY. An autopsy case of sarcoidosis in the intestinal canal. Bull Osaka Med School 1972;18:48-55.

2 Clague RB. Sarcoidosis or Crohn's disease? $\mathrm{Br}$ Med $\mathrm{f}$ 1972;3:804.

3 Sprague R, Harper P, McLain S, Trainer T, Beeken W Disseminated gastrointestinal sarcoidosis. Case report and review of the literature. Gastroenterology 1984;87:421-5.

4 Tinker MA, Viswanathan B, Laufer H, Margolis IB. Acute appendicitis and pernicious anemia as complications of gastrointestinal sarcoidosis. Am f Gastroenterol 1984;79:868-72.

5 Bulger K, O'Riordan M, Purdy S, O'Brien J, Lennon J. Gastrointestinal sarcoidosis resembling Crohn's disease. Am $\mathcal{F}$ Gastroenterol 1988;83:1415-7.

6 Rauf A, Davis P, Levendoglu H. Sarcoidosis of the small intestine. Am f Gastroenterol 1988;83:187-9.

7 James DG. Miscellaneous involvement. In: James DG, Williams WJ, eds. Sarcoidosis and other granulomatous disorders. Problems in internal medicine. Vol 24. Philadelphia: disorders. Problems in internal medic

8 Tweedie JH, McCann BG. Metastatic Crohn's disease of thigh and forearm. Gut 1984;25:213-14.

9 Lemann M, Messing B, D'Agay F, Modigliani R. Crohn's disease with respiratory tract involvement. Gut 1987;28:1669-72.

10 Siltzbach LE, Vieria LOBD, Topilsky M, Janowitz HD. Is there Kveim responsiveness in Crohn's disease? Lancet 1971;ii:634-5.

\title{
Immunoperoxidase techniques and histology in the diagnosis of rhabdomyolysis related acute renal failure
}

\author{
P N Cooper, J Tapson, A R Morley
}

\author{
Department of \\ Histopathology, Royal \\ Victoria Infirmary, \\ Newcastle upon Tyne \\ P N Cooper \\ A R Morley \\ Department of Renal \\ Medicine, Freeman \\ Hospital, Newcastle \\ upon Tyne \\ J Tapson \\ Correspondence to: \\ Dr P N Cooper, \\ Department of Pathology, \\ Royal Victoria Infirmary, \\ Queen Victoria Road, \\ Newcastle upon Tyne, \\ NE1 4LP \\ Accepted for publication \\ 24 February 1992
}

\begin{abstract}
A case of rhabdomyolysis associated acute renal failure (RM-ARF) occurring as a result of strenuous exercise is presented. Diagnostic renal biopsy was performed. The histological appearances, combined with immunoperoxidase staining for myoglobin, allowed a positive diagnosis of RM-ARF to be made and excluded the possibility of glomerulonephritis. The patient recovered completely after a stormy clinical course.
\end{abstract}

Rhabdomyolysis can be the result of many diverse factors including direct muscle injury, excessive muscle activity, ischaemia, immunological disease, metabolic disorders, drugs, toxins, infection and genetic disorders. In two large series rhabdomyolysis associated acute renal failure (RM-ARF) represented between
$5 \%$ and $8.6 \%$ of cases of acute renal failure. $^{12}$

In the absence of trauma the clinical symptoms and signs of muscle damage are unreli$\mathrm{able}^{23}$ and diagnosis depends on finding raised serum creatine phosphokinase (CPK) activity and o-toluidine positive urine and golden coloured granular casts on microscopical examination of the urine. The prognosis of RM-ARF is good provided prompt treatment is given. ${ }^{1}$

We report a case of exertion related RMARF in a 17 year old soldier who developed RM-ARF following exercise.

\section{Case report}

A 17 year old private collapsed after running for 7.5 miles wearing army boots and a full backpack on a day in October during which the temperature reached a maximum of $13.9^{\circ} \mathrm{C}$. 